\begin{tabular}{|c|l|}
\hline Title & The Rohlin property for automorphisms of UHF algebras \\
\hline Author(s) & Kishimoto, A. \\
\hline Citation & Hokkaido University Preprint Series in Mathematics, 288, 1-15 \\
\hline Issue Date & 1995-4-1 \\
\hline DOI & 10.14943/83435 \\
\hline Doc URL & http://hdl.handle.net/2115/69039 \\
\hline Type & bulletin (article) \\
\hline File Information & pre288.pdf \\
\hline
\end{tabular}

Instructions for use 
The Rohlin property for automorphisms of UHF algebras

\section{Akitaka Kishimoto}

Series $\sharp 288$. April 1995 


\section{HOKKAIDO UNIVERSITY \\ PREPRINT SERIES IN MATHEMATICS}

\#264 J. Wierzbicki, On Commutativity of Diagrams of Type $\mathrm{II}_{1}$ Factors, 26 pages. 1994.

$\sharp 265$ N. Hayashi, T. Ozawa, Schrödinger Equations with nonlinearity of integral type, 12 pages. 1994.

$\sharp 266$ T. Ozawa, On the resonance equations of long and short waves, 8 pages. 1994.

$\sharp 267$ T. Mikami, A sufficient condition for the uniqueness of solutions to a class of integro-differential equations, 9 pages. 1994.

$\sharp 268 \quad$ Y. Giga, Evolving curves with boundary conditions, 10 pages. 1994.

$\sharp 269$ A. Arai, Operator-theoretical analysis of representation of a supersymmetry algebra in Hilbert space, 12 pages. 1994.

$\sharp 270$ A. Arai, Gauge theory on a non-simply-connected domain and representations of canonical commutation relations, 18 pages. 1994 .

$\sharp 271$ S. Jimbo, Y. Morita and J. Zhai, Ginzburg landau equation and stable steady state solutions in a non-trivial domain, 17 pages. 1994.

\#272 S. Izumiya, A. Takiyama, A time-like surface in Minkowski 3-space which contains light-like lines, 7 pages. 1994.

\#273 K. Tsutaya, Global existence of small amplitude solutions for the Klein-Gordon-Zakharov equations, 11 pages. 1994.

$\sharp 274$ H. Kubo, On the critical decay and power for semilinear wave equations in odd space dimensions, 22 pages. 1994.

$\sharp 275$ N. Terai, T. Hibi, Alexander duality theorem and second Betti numbers of Stanley-Reisner rings, 2 pages. 1995.

$\sharp 276$ N. Terai, T. Hibi, Stanley-Reisner rings whose Betti numbers are independent of the base field, 12 pages. 1995.

$\sharp 277$ N. Terai, T. Hibi, Computation of Betti numbers of monomial ideals associated with cyclic polytopes, 11 pages. 1995.

$\sharp 278$ N. Terai, T. Hibi, Computation of Betti numbers of monomial ideals associated with stacked polytopes, 8 pages. 1995.

$\sharp 279$ N. Terai, T. Hibi, Finite free resolutions and 1-skeletons of simplicial $(d-1)$-spheres, 3 pages. 1995.

\#280 N. Terai, T. Hibi, Monomial ideals and minimal non-faces of Cohen-Macaulay complexes, 6 pages. 1995.

\#281 A. Arai, N. Tominaga, Analysis of a family of strongly commuting self-adjoint operators with applications to perturbed d'Alembertians and the external field problem in quantum field theory, 44 pages. 1995.

$\sharp 282$ T. Mikami, Asymptotic behavior of the first exit time of randomly perturbed dynamical systems with a repulsive equilibrium point, 29 pages. 1995.

$\sharp 283 \quad$ K. Iwata, J. Schäfer, Markov property and cokernels of local operators, 17 pages. 1995.

$\sharp 284$ T. Nakazi, M. Yamada, Riesz's Functions In Weighted Hardy And Bergman Spaces, 20 pages. 1995.

$\sharp 285$ K. Hidano, K. Tsutaya, Scattering theory for nonlinear wave equations in the invariant Sobolev space, 32 pages. 1995.

$\sharp 286$ A. Arai, Strong coupling limit of the zero-energy-state density of the Dirac-Weyl operator with a singular vector potential, 8 pages. 1995 .

$\sharp 287$ T. Nakazi, Factorizations of outer functions and extremal problems, 15 pages. 1995. 


\title{
The Rohlin property for automorphisms of UHF algebras
}

\author{
Akitaka Kishimoto
}

February 1995

\begin{abstract}
For an automorphism $\alpha$ of a UHF algebra it is shown that $\alpha$ has the Rohlin property if and only if $\alpha^{m}$ is uniformly outer for any $m \neq 0$. It is also shown that the automorphisms of a UHF algebra with the Rohlin property are outer conjugate with each other.
\end{abstract}

\section{Introduction}

Let $\alpha$ be an automorphism of a unital $C^{*}$-algebra $A$. First we recall some definitions $[13,6,17,15]$.

Definition 1.1 The automorphism $\alpha$ has the Rohlin property if for any $k \in \mathbf{N}$ there are positive integers $k_{1}, \ldots, k_{m} \geq k$ satisfying the following condition: For any finite subset $F$ of $A$ and $\epsilon>0$ there are projections $e_{i, j} ; i=1, \ldots, m, j=0, \ldots, k_{i}-1$ in $A$ such that

$$
\begin{aligned}
& \sum_{i=1}^{m} \sum_{j=0}^{k_{i}-1} e_{i, j}=1, \\
& \left\|\alpha\left(e_{i, j}\right)-e_{i, j+1}\right\|<\epsilon, \\
& \left\|\left[x, e_{i, j}\right]\right\|<\epsilon
\end{aligned}
$$

for $i=1, \ldots, m, j=0, \ldots, k_{i}-1$, and $x \in F$ where $e_{i, k_{i}}=e_{i, 0}$.

Definition 1.2 The automorphism $\alpha$ is uniformly outer if for any $a \in A$, any projection $p \in A$, and any $\epsilon>0$, there are a finite number of projections $p_{1}, \ldots, p_{n}$ in $A$ such that

$$
\begin{aligned}
& p=\sum_{i=1}^{n} p_{i}, \\
& \left\|p_{i} a \alpha\left(p_{i}\right)\right\|<\epsilon, i=1, \ldots, n .
\end{aligned}
$$


If $\alpha$ has the Rohlin property then $\alpha^{m}$ is uniformly outer for any $m \neq 0$. The converse is shown for Cuntz algebras $A=O_{n}$ in [15]. In this note we shall show the converse for UHF algebras (and for certain unital simple AF algebras). This generalizes the results on shifts on UHF algebras $M_{n \infty}[6,15]$ and on quasi-free automorphisms of the CAR algebras [4]. Note that if $A$ is UHF, the property that $\alpha^{m}$ is uniformly outer for any $m \neq 0$ is equivalent to that the crossed product $A \times_{\alpha} \mathbf{Z}$ has a unique tracial state [15]. To be more precise, the former condition implies, by [15], Lemma 4.3, that any tracial state of $A \times{ }_{\alpha} \mathbf{Z}$ is invariant under the dual action $\hat{\alpha}$, and hence that $A \times_{\alpha} \mathbf{Z}$ has a unique tracial state; which implies in turn, by (the proof of) [15], Lemma 4.4, that $\bar{\alpha}^{m}$ is outer for any $m \neq 0$, where $\bar{\alpha}$ denotes the extension of $\alpha$ to an automorphism of the weak closure of $A$ in the tracial representation; which implies, by [15], Theorem 4.5, the condition on $\alpha$ we started with.

If an automorphism $\alpha$ of a UHF algebra $A$ has the Rohlin property, then for any $\epsilon>0$ there is a unitary $u \in A$ such that $\|u-1\|<\epsilon$ and $\operatorname{Ad} u \circ \alpha$ is of infinite tensor product type $[18,4]$. (This follows from the proof of [4], Theorem 1.7, which asserts the same conclusion for certain UHF algebras based on a special form of Rohlin property. Adopting the present form of Rohlin property as suggested by Rørdam [17], the conclusion holds for any UHF algebras.) Furthermore $A \times_{\alpha} \mathbf{Z}$ is approximately divisible [3] and is isomorphic to a unique simple AT algebra independent of $\alpha$ (as far as $\alpha$ has the Rohlin property) [4]. ( $K_{*}\left(A \times_{\alpha} \mathbf{Z}\right) \cong K_{0}(A)$ by the Pimsner-Voiculescu exact sequence [1]; $A \times_{\alpha} \mathbf{Z}$ is an inductive limit of $C^{*}$-algebras of the form $C(\mathbf{T}) \otimes M_{n}$, called an AT algebra; $A \times_{\alpha} \mathbf{Z}$ has real rank zero [2]: These properties imply the above assertion by Elliott's theory [12].) Note that if we just assume that any non-zero power of $\alpha$ is outer (instead of uniformly outer), we cannot expect in general the closeness, in norm, of $\alpha$ to an automorphism of infinite tensor product type (cf. [8]). Summing up such results we obtain the following result; the corresponding result for quasi-free automorphisms of the CAR algebra is given in [4], Theorem 1.1:

Theorem 1.3 Let $A$ be a UHF algebra, i.e., the inductive limit of an increasing sequence $\left\{M_{n_{1}} \otimes \cdots \otimes M_{n_{k}}\right\}$ of full matrix algebras with natural embeddings. If $\alpha$ is an automorphism of $A$, the following conditions are equivalent:

1. $\alpha$ has the Rohlin property.

2. $\alpha^{m}$ is uniformly outer for any $m \neq 0$.

3. $A \times_{\alpha} \mathbf{Z}$ has a unique tracial state.

4. $A \times_{\alpha} \mathbf{Z}$ has real rank zero.

5. There exists a unitary $u \in A$ such that $(A, \operatorname{Ad} u \circ \alpha)$ is isomorphic to

$$
\left(\otimes_{k=1}^{\infty} M_{m_{k}}, \otimes_{k=1}^{\infty} \operatorname{Ad} u_{k}\right)
$$

where $u_{k}$ is a unitary of $M_{m_{k}}$ and the eigenvalues of $\otimes_{k=l}^{\infty} u_{k}$ are uniformly distributed for any $l \in \mathbf{N}$. 
6. $A \times_{\alpha} \mathbf{Z}$ is the inductive limit of an increasing sequence $\left\{B_{k}\right\}$ of $C^{*}$-algebras such that

$$
B_{k} \equiv C(\mathbf{T}) \otimes M_{n_{1}} \otimes \cdots \otimes M_{n_{k}} \cong M_{n_{k}}\left(B_{k-1}\right)
$$

and the embedding of $B_{k}$ into $B_{k+1}$ is given by

$$
f \mapsto f \oplus \beta_{\theta}(f) \oplus \beta_{2 \theta}(f) \oplus \cdots \oplus \beta_{\left(n_{k+1}-1\right) \theta}(f),
$$

where $\theta$ is irrational and $\beta_{\theta}(f)(t)=f(t-\theta)$ for $f \in B_{k} \cong C\left(\mathbf{T}, M_{n_{1}} \otimes \cdots \otimes M_{n_{k}}\right)$.

When (1) holds, (6) is obtained by choosing a special $\alpha$ by using the uniqueness of $A \times_{\alpha} \mathrm{Z}$ mentioned above; (6) implies (3) (by an easy computation) and (4) (by [2]). See Section 5 for details of (5); (5) implies (1) by the condition of uniform distribution (Lemma 5.2). We have noted before the theorem that $(5) \Leftarrow(1) \Rightarrow(2) \Leftrightarrow(3)$. We shall show that (4) implies (3). A $C^{*}$-algebra has real rank zero if and only if the self-adjoint elements with finite spectra are dense in the set of all self-adjoint elements [7]. Hence if (4) holds, the linear span of projections is dense in $A \times_{\alpha} \mathbf{Z}$ and so any tracial state on $A \times_{\alpha} \mathbf{Z}$ is invariant under the dual action of $\hat{\mathbf{Z}} \cong \mathrm{T}$ which is connected. Since $A$ has only one tracial state, this shows that (4) implies (3). Sections $2-4$ are devoted to the proof of the remaining implication $(2) \Rightarrow(1)$.

In Section 2 we give a simple main technical lemma concerning the shift automorphism on the compact operators on $l^{2}(\mathbf{Z})$. This asserts that this automorphism has a kind of Rohlin property. In Section 3 using Connes' result [9] on the Rohlin property for automorphisms of the injective type $\mathrm{II}_{1}$ factor, we show that we can simulate a system as in Section 2 in certain systems $(A, \alpha)$ if the automorphism $\alpha$ satisfies the property that $\alpha^{m}$ is uniformly outer for any $m \neq 0$. If the $C^{*}$-algebra $A$ is UHF, this simulation can be done in the relative commutant of any finite-dimensional subalgebra. Combining these results we conclude, in Section 4, that $\alpha$ has the approximate Rohlin property [5]. Using the methods given in [15] we then conclude that $\alpha$ has the Rohlin property.

In Section 5 we shall show (cf. [14]):

Theorem 1.4 Let $\alpha$ and $\beta$ be automorphisms of a UHF algebra $A$. If $\alpha$ and $\beta$ have the Rohlin property, then for any $\epsilon>0$ there exists an automorphism $\gamma$ of $A$ such that $\left\|\alpha-\gamma \circ \beta \circ \gamma^{-1}\right\|<\epsilon$.

The conclusion of this theorem is equivalent to saying that $\alpha$ and $\beta$ are outer conjugate. Since an automorphism of a unital simple $C^{*}$-algebra is inner if it is close to the identity in norm, the outer conjugacy follows from the conclusion. If $\operatorname{Ad} u \circ \alpha=\gamma \circ \beta \circ \gamma^{-1}$ and $\left\|u-v^{*} \alpha(v)\right\|<\epsilon$ for some unitary $v \in A$, then $\left\|\alpha-\operatorname{Ad} v \circ \gamma \circ \beta \circ \gamma^{-1} \circ \operatorname{Ad} v^{*}\right\|<2 \epsilon$ follows; the existence of the $v$ above follows from the Rohlin property by [13]. Hence the uniqueness of $A \times_{\alpha} \mathbf{Z}$ asserted by using Elliott's result [12] is also a consequence of this theorem.

In Section 6 we shall show by similar methods that an automorphism $\alpha$ of a simple non-commutative torus which comes from the gauge action has the Rohlin property if any non-zero power of $\alpha$ is outer. Since the crossed product by $\alpha$ depends on $\alpha$ in general [11], a result like the above theorem cannot hold in this case. 


\section{Shift on $l^{2}(\mathbf{Z})$}

Let $\left\{E_{i j} ; i, j \in \mathbf{Z}\right\}$ be matrix units:

$$
\left(E_{i j}\right)^{*}=E_{j i}, E_{i j} E_{k l}=\delta_{j k} E_{i l}
$$

Let $K$ be the closed linear span of these $E_{i j}$ with unique $\mathrm{C}^{*}$-norm; $K$ is the compact operators on $l^{2}(\mathbf{Z})$ by identifying $E_{i i}$ with the one-dimensional projection onto the functions supported by $\{i\} \subset \mathbf{Z}$. Define an automorphism $\sigma$ of $K$ by $\sigma\left(E_{i, j}\right)=E_{i+1, j+1}$. For any $N \in \mathbf{N}$ let $P_{N}=\sum_{i=0}^{N-1} E_{i i}$.

Lemma 2.1 Let $K, \sigma, P_{N}$, etc be as above. For any $\epsilon>0$ and $n \in \mathbf{N}$ there exist $N \in \mathbf{N}$ and projections $e_{0}, e_{1}, \ldots, e_{n-1}$ in $K$ such that

$$
\begin{aligned}
& \sum_{i=0}^{n-1} e_{i} \leq P_{N} \\
& \left\|\sigma\left(e_{i}\right)-e_{i+1}\right\|<\epsilon, i=0, \ldots, n-1, \\
& \frac{n \operatorname{dim} e_{0}}{N}>1-\epsilon
\end{aligned}
$$

where $e_{n}=e_{0}$.

Proof. We shall choose $k, l \in \mathbf{N}$ such that $1 \ll k \ll l$. Define

$$
\begin{aligned}
f=\quad & \sum_{m=1}^{k-1}\left\{\frac{m}{k} E_{n m, n m}+\frac{k-m}{k} E_{n(m+k+l), n(m+k+l)}\right. \\
& \left.+\frac{\sqrt{m(k-m)}}{k} E_{n m, n(m+k+l)}+\frac{\sqrt{m(k-m)}}{k} E_{n(m+k+l), n m}\right\} \\
& +\sum_{m=k}^{k+l} E_{n m, n m} .
\end{aligned}
$$

Then $f$ is a projection such that

$$
f \leq \sum_{m=1}^{2 k+l-1} E_{n m, n m}
$$

and the dimension of $f$ is equal to $k+l$. For $m=0, \ldots, n-1$ let $e_{m}=\sigma^{m-n}(f)$. Let $N=n(2 k+l-1)$. Then $\left\{e_{m} ; m=0, \ldots, n-1\right\}$ are mutually orthogonal projections of $K$ such that $e_{0}+\cdots+e_{n-1} \leq P_{N}$. Since $\sigma\left(e_{n-1}\right)-e_{0}=f-e_{0}$ is equal to

$$
\begin{aligned}
& -\frac{1}{k} \sum_{m=0}^{k-1} E_{n m, n m}+\frac{1}{k} \sum_{m=0}^{k-1} E_{n(m+k+l), n(m+k+l)} \\
& +\sum_{m=0}^{k-1} \frac{1}{k}[\sqrt{m(k-m)}-\sqrt{(m+1)(k-m-1)}]\left\{E_{n m, n(m+k+l)}+E_{n(m+k+l), n m}\right\}
\end{aligned}
$$


and for $m=0, \ldots, k-1$

$$
|\sqrt{m(k-m)}-\sqrt{(m+1)(k-m-1)}|<\sqrt{k}
$$

we have that

$$
\left\|\sigma\left(e_{n-1}\right)-e_{0}\right\|<\frac{1}{k}+\frac{1}{\sqrt{k}} .
$$

Hence if $k$ is so large that $k^{-1}+k^{-1 / 2}<\epsilon$, then we have that $\left\|\sigma\left(e_{i}\right)-e_{i+1}\right\|<\epsilon$ for $i=0, \ldots, n-1$ with $e_{n}=e_{0}$. Since

$$
\frac{n \operatorname{dim} e_{0}}{N}=\frac{k+l}{2 k+l-1}
$$

if $l$ is so large that

$$
\epsilon>\frac{k-1}{2 k+l-1}
$$

then we have that

$$
\frac{n \operatorname{dim} e_{0}}{N}>1-\epsilon
$$

This concludes the proof.

\section{Simulation}

We consider a certain class of AF algebras which includes UHF algebras. Let $A$ be a unital infinite-dimensional simple AF algebra. Note that $K_{0}(A)$ is of finite rank $r$ and has no infinitesimal elements if and only if $K_{0}(A)$ is order isomorphic to a dense subgroup of $\mathbf{R}^{d}$ for some $d \leq r$, provided with the relative strict order [10]. (The strict order on $\mathbf{R}^{d}$ is defined as follows: $a \leq b$ if $a=b$ or $a_{i}<b_{i}$ for $i=1, \ldots, d$.) In this case $A$ has $d$ extreme tracial states.

Lemma 3.1 Let $A$ be a unital simple $A F$ algebra such that $K_{0}(A)$ is of finite rank and has no infinitesimal elements. Let $\alpha$ be an automorphism of $A$ such that $\alpha^{m}$ is uniformly outer for any $m \neq 0$. For any $m \in \mathbf{N}, \epsilon>0$, and any finite-dimensional $C^{*}$-subalgebra $B$ of $A$ there exists an orthogonal family $\left\{e_{i} ; i=0, \ldots, m\right\}$ of projections in $A \cap B^{\prime}$ such that

$$
\begin{aligned}
& \left\|\alpha\left(e_{i}\right)-e_{i+1}\right\|<\epsilon, i=0, \ldots, m-1 \\
& {\left[e_{0}\right]=\left[e_{1}\right]=\cdots=\left[e_{m}\right]} \\
& m[1] \leq(m+k)(m+1)\left[e_{0}\right]
\end{aligned}
$$

where [.] denotes the equivalence class in $K_{0}\left(A \cap B^{\prime}\right)$ and $k$ is the period of $\alpha_{*}$ on $K_{0}(A)$. 
Proof. Let $\left\{\tau_{1}, \ldots, \tau_{d}\right\}$ be the set of extreme tracial states of $A$. For each $i=1, \ldots, d$ let $k_{i}$ be the minimal positive integer such that $\tau_{i} \circ \alpha^{k_{i}}=\tau_{i}$ and let $k$ be the least common multiple of $k_{1}, \ldots, k_{d}$. Then $k$ is the minimal positive integer such that $\tau \circ \alpha^{k}=\tau$ for any tracial state $\tau$ on $A$ or equivalently $\alpha_{*}^{k}=i d$ on $K_{0}(A)$.

Let $\rho=\bigoplus_{i=1}^{d} \pi_{\tau_{i}}$. Then there is a unique automorphism $\bar{\alpha}$ of $\rho(A)^{\prime \prime}$ such that $\bar{\alpha} \circ \rho=$ $\rho \circ \alpha$. Since $\pi_{\tau_{i}}(A)^{\prime \prime}$ is an injective type $\mathrm{II}_{1}$ factor and any non-zero power of $\bar{\alpha}^{k_{i}}$ on $\pi_{\tau_{i}}(A)^{\prime \prime}$ is outer [15], Theorem 4.5, we can apply [9] to $\bar{\alpha}^{k_{i}} \mid \pi_{\tau_{i}}(A)^{\prime \prime}$ to conclude that $\bar{\alpha}^{k_{i}} \mid \pi_{\tau_{i}}(A)^{\prime \prime}$ has the (von Neumann version of) Rohlin property. Thus we have, for any $l \in \mathbf{N}$, a central sequence $\left\{\left(E_{0}^{(j)}, \ldots, E_{k_{i} l-1}^{(j)}\right)\right\}$ of orthogonal families of projections in $\oplus_{s=0}^{k_{i}-1} \pi_{r_{i} \circ \alpha^{s}}(A)^{\prime \prime}$ such that

$$
\begin{aligned}
& \sum_{i=0}^{k_{i} l-1} E_{i}^{(j)} \rightarrow 1 \\
& \bar{\alpha}\left(E_{i}^{(j)}\right)-E_{i+1}^{(j)} \rightarrow 0, i=0, \ldots, k_{i} l-1,
\end{aligned}
$$

strongly as $j \rightarrow \infty$, where $E_{k_{i} l}^{(j)}=E_{0}^{(j)}$. Since $\rho(A)^{\prime \prime}$ is the finite direct sum of such von Neumann algebras, we can conclude that for any $l \in \mathbf{N}$ there exists a central sequence $\left\{\left(E_{0}^{(j)}, \ldots, E_{k l-1}^{(j)}\right)\right\}$ of orthogonal families of projections in $\rho(A)^{\prime \prime}$ such that

$$
\begin{aligned}
& \sum_{i=0}^{k l-1} E_{i}^{(j)} \rightarrow 1, \\
& \bar{\alpha}\left(E_{i}^{(j)}\right)-E_{i+1}^{(j)} \rightarrow 0, i=0, \ldots, k l-1,
\end{aligned}
$$

strongly as $j \rightarrow \infty$, where $E_{k l}^{(j)}=E_{0}^{(j)}$.

Then we can replace $\left\{E_{i}^{(j)}\right\}$ by a central sequence $\left\{e_{i}^{(j)}\right\}$ of orthogonal families of projections in $A$ such that

$$
\begin{aligned}
& \rho\left(\sum_{i=0}^{k l-1} e_{i}^{(j)}\right) \rightarrow 1, \\
& \left\|\alpha\left(e_{i}^{(j)}\right)-e_{i+1}^{(j)}\right\| \rightarrow 0, i=0, \ldots, k l-2
\end{aligned}
$$

as $j \rightarrow \infty$. (See [15], Section 4 for details.) Actually what we do is as follows: First find a central sequence $\left\{e_{j}\right\}$ of projections of $A$ such that $\rho\left(e_{j}\right)-E_{0}^{(j)} \rightarrow 0$ strongly. Second, noting that $\rho\left(e_{j}\left(\sum_{s=1}^{k l-1} \alpha^{s}\left(e_{j}\right)\right) e_{j}\right) \rightarrow 0$, find a subprojection $p_{j}$ of $e_{j}$ such that $\left\{p_{j}\right\}$ is a central sequence and

$$
\begin{aligned}
& \left\|p_{j}\left(\sum_{s=1}^{k l-1} \alpha^{s}\left(e_{j}\right)\right) p_{j}\right\| \rightarrow 0, \\
& \rho\left(e_{j}-p_{j}\right) \rightarrow 0 .
\end{aligned}
$$

The condition of centrality can be satisfied by assuming that the algebra generated by $e_{j}, \alpha\left(e_{j}\right), \ldots, \alpha^{k l-1}\left(e_{j}\right)$ lies in the relative commutant of a prescribed finite-dimensional 
$C^{*}$-subalgebra and then by choosing $p_{j}$ from this commutant. Then the projections $p_{j}, \alpha\left(p_{j}\right), \ldots, \alpha^{k l-1}\left(p_{j}\right)$ will be nearly orthogonal, and hence we can construct, by a standard technique, an orthogonal family $\left\{f_{0}^{(j)}, \ldots, f_{k l-1}^{(j)}\right\}$ of projections such that $f_{s}^{(j)} \approx$ $\alpha^{s}\left(p_{j}\right)$. Furthermore we can assume that all $f_{s}^{(j)}$ are in $A \cap B^{\prime}$.

Given $m \in \mathbf{N}$ let $m_{0} \geq m$ be the smallest integer such that $m_{0} \equiv 0 \bmod k$. Let $l=m_{0}+1$ and

$$
e_{i}^{(j)}=\sum_{s=0}^{k-1} f_{i+s\left(m_{0}+1\right)}, i=0, \ldots, m .
$$

Then $\left\{e_{i}^{(j)} ; i=0, \ldots, m\right\}$ is a central sequence in $j$ and $\left[e_{i}^{(j)}\right]$ is independent of $i$ in $K_{0}\left(A \cap B^{\prime}\right)$ for a sufficiently large $j$ by Lemma 3.2 below. It also follows that

$$
\begin{aligned}
& e^{(j)}=\sum_{i=0}^{m} e_{i}^{(j)} \leq 1, \\
& \left\|\alpha\left(e_{i}^{(j)}\right)-e_{i+1}^{(j)}\right\| \rightarrow 0, i=0, \ldots, m-1, \\
& \tau_{s}\left(e^{(j)}\right) \rightarrow \frac{m+1}{m_{0}+1}, s=1, \ldots, d .
\end{aligned}
$$

Since $\tau_{s}$ is factorial, it follows that for any minimal central projection $E$ of $B$,

$$
\tau_{s}\left(e^{(j)} E\right) \rightarrow \frac{m+1}{m_{0}+1} \tau_{s}(E) .
$$

Since the traces $\tau_{1}, \ldots, \tau_{d}$ on $\left(A \cap B^{\prime}\right) E$ determine the order of the dimension group of $\left(A \cap B^{\prime}\right) E$, which is order isomorphic to $K_{0}(A)$, it follows that

$$
\left(m_{0}+1\right)\left[e^{(j)}\right] \geq m[1]
$$

in $K_{0}\left(A \cap B^{\prime}\right)$ if $j$ is sufficiently large. Since $m_{0} \leq m+k-1$, this concludes the proof.

Lemma 3.2 In the situation of Lemma 3.1 let $\left\{p_{j}\right\}$ be a central sequence of projections in $A$ and let $\beta=\alpha^{k l}$ for some $l \in \mathbf{N}$. Then there is a central sequence $\left\{x_{j}\right\}$ in $A$ such that $x_{j}^{*} x_{j}=p_{j}$ and $x_{j} x_{j}^{*}=\beta\left(p_{j}\right)$.

Proof. Let $D$ be a finite-dimensional subalgebra of $A$ and let $v$ be a unitary of $A$ such that $\beta|D=\operatorname{Ad} v| D$. Then for a sufficiently large $j, p_{j}$ almost commutes with $D$ and $\beta\left(p_{j}\right)$ is close to $\operatorname{Ad} v^{*} \circ \beta\left(p_{j}\right)$. Since $\operatorname{Ad} v^{*} \circ \beta \mid D=i d$, it follows that there is a unitary $u_{j} \in A \cap D^{\prime}$ such that $\operatorname{Ad} v^{*} \circ \beta\left(p_{j}\right) \approx \operatorname{Ad} u_{j}\left(p_{j}\right)$. Thus we can find $x_{j}$ with $x_{j}^{*} x_{j}=p_{j}, x_{j} x_{j}^{*}=\beta\left(p_{j}\right)$ in a small neighbourhood of $u_{j} p_{j}$, which implies that $x_{j}$ almost commutes with $D$. Since $D$ is arbitrary, we obtain the conclusion. 


\section{The Rohlin property}

Theorem 4.1 Let $A$ be a unital simple $A F$ algebra such that $K_{0}(A)$ is of finite rank and has no infinitesimal elements. Let $\alpha$ be an automorphism of $A$. If $\alpha$ satisfies the property that $\alpha^{m}$ is uniformly outer for any $m \neq 0$, then $\alpha$ has the Rohlin property.

We note the following consequence obtained in [18]: In the above theorem further suppose that $\alpha_{*}=i d$ on $K_{0}(A)$. Then for any $\epsilon>0$ there are a unitary $u \in A$ and an increasing sequence $\left\{A_{n}\right\}$ of finite-dimensional subalgebras of $A$ such that $\|u-1\|<\epsilon$, $\cup A_{n}$ is dense in $A$, and Ad $u \circ \alpha\left(A_{n}\right)=A_{n}$ for all $n$.

Before giving the proof we introduce the following definition [5].

Definition 4.2 An automorphism $\alpha$ of a unital AF algebra $A$ has the approximate Rohlin property if for any $m, n \in \mathbf{N}$, any $\epsilon>0$, and any finite-dimensional $C^{*}$-subalgebra $B$ of $A$ there is an orthogonal family $\left\{e_{i} ; i=0, \ldots, m-1\right\}$ of projections in $A \cap B^{\prime}$ such that

$$
\begin{aligned}
& \left\|\alpha\left(e_{i}\right)-e_{i+1}\right\|<\epsilon, i=0, \ldots, m-1, \\
& {\left[e_{0}\right]=\left[e_{1}\right]=\cdots=\left[e_{m-1}\right]} \\
& {\left[e_{0}\right] \geq n\left[1-\sum_{i=0}^{m-1} e_{i}\right]}
\end{aligned}
$$

where $[\cdot]$ denotes the equivalence class in $K_{0}\left(A \cap B^{\prime}\right)$ and $e_{m}=e_{0}$.

Theorem 4.1 follows from the following two lemmas.

Lemma 4.3 Under the situation of Theorem 4.1 suppose that $\alpha^{m}$ is uniformly outer for any $m \neq 0$. Then $\alpha$ has the approximate Rohlin property.

Proof. This follows from 2.1 and 3.1. Let $k$ be the period of $\alpha_{*}$ on $K_{0}(A)$ as in the proof of 3.1. Let $m \in \mathbf{N}, \epsilon>0$, and $B$ a finite-dimensional $C^{*}$-subalgebra of $A$. By 2.1 one finds $N \in \mathbf{N}$ and projections $e_{0}, \ldots, e_{m-1}$ in $K$ such that

$$
\begin{aligned}
& e_{0}+\cdots+e_{m-1} \leq P_{N}, \\
& \left\|\sigma\left(e_{i}\right)-e_{i+1}\right\|<\epsilon, \\
& \frac{m \operatorname{dim} e_{0}}{N}>1-\epsilon
\end{aligned}
$$

where $e_{m}=e_{0}$. By 3.1 one finds $N+1$ projections $p_{0}, \ldots, p_{N}$ in $A \cap B^{\prime}$ such that

$$
\begin{aligned}
& p_{0}+\cdots+p_{N} \leq 1 \\
& \left\|\alpha\left(p_{i}\right)-p_{i+1}\right\|<\epsilon / N, i=0, \ldots, N-1
\end{aligned}
$$


and $\left[p_{0}\right]=\cdots=\left[p_{N}\right], N[1] \leq(N+k)(N+1)\left[p_{0}\right]$ in $K_{0}\left(A \cap B^{\prime}\right)$. Then there is a unitary $u \in A$ such that $\|u-1\|<4 \epsilon$ and $\operatorname{Ad} u \circ \alpha\left(p_{i}\right)=p_{i+1}$ for $i=0, \ldots, N-1$. Let $w$ be a partial isometry in $A \cap B^{\prime}$ such that $w^{*} w=p_{0}$ and $w w^{*}=p_{1}$. Let $\alpha_{1}=\operatorname{Ad} u \circ \alpha$ and let $C_{1}$ (resp. $C$ ) be the $C^{*}$-algebra generated by $w, \alpha_{1}(w), \ldots, \alpha_{1}^{N-2}(w)$ (resp. and $\alpha_{1}^{N-1}(w)$ ). Then $M_{N} \cong C_{1} \subset C \cong M_{N+1}$. Define a homomorphism $\Phi$ of $C$ into $K$ (as in 2.1) by $\Phi\left(\alpha_{1}^{i}(w)\right)=E_{i+1, i}$. Then $\Phi$ satisfies that $\sigma \circ \Phi\left|C_{1}=\Phi \circ \alpha_{1}\right| C_{1}$ and $\Phi\left(C_{1}\right)=P_{N} K P_{N}$. Thus, denoting $\Phi^{-1}\left(e_{i}\right)$ by $e_{i}$, we obtain projections $e_{0}, \ldots, e_{m-1}$ in $C_{1} \subset A \cap B^{\prime}$ such that

$$
\begin{aligned}
& e_{0}+\cdots+e_{m-1} \leq 1, \\
& \left\|\alpha\left(e_{i}\right)-e_{i+1}\right\|<9 \epsilon, i=0, \ldots, m-1, \\
& m\left[e_{0}\right] \geq \frac{N^{2}}{(N+k)(N+1)}(1-\epsilon)[1]
\end{aligned}
$$

where $e_{m}=e_{0}$ and the last inequality is in $K_{0}\left(A \cap B^{\prime}\right) \otimes \mathbf{R}$. For a sufficiently large $N$ and a sufficiently small $\epsilon>0$ we can conclude the proof.

Lemma 4.4 Under the situation of Theorem 4.1 suppose that $\alpha$ has the approximate Rohlin property. Then $\alpha$ has the Rohlin property.

Proof. This follows by the arguments in the proof of Theorem 2.1 of [15]. We just outline the proof. The approximate Rohlin property is not good enough only because

$$
e=\sum_{i=0}^{m} e_{i}
$$

is not 1 , where $e_{i}$ 's are as in Definition 4.2. But $1-e$ is small compared with $e_{0}$ (if $n$ is large), which is the fact we shall use below. Assuming $\epsilon>0$ is sufficiently small, let us assume that $\alpha\left(e_{i}\right)=e_{i+1}$ and so $\alpha^{m}\left(e_{0}\right)=e_{0}$. We use the approximate Rohlin property for $\left(e_{0} A e_{0}, \alpha^{m}\right)$ to obtain projections $p_{1}, \ldots, p_{n}$ in $A \cap B^{\prime}$ such that

$$
\begin{aligned}
& p_{1}+\cdots+p_{n} \leq e_{0}, \\
& \alpha^{m}\left(p_{i}\right) \approx p_{i+1}, i=1, \ldots, n-1,
\end{aligned}
$$

and $\left[p_{1}\right]=[1-e]$ in $K_{0}\left(A \cap B^{\prime}\right)$. Let $v$ be a partial isometry in $A \cap B^{\prime}$ such that $v^{*} v=1-e$ and $v v^{*}=p_{1}$ and let

$$
w^{\prime}=n^{-1 / 2} \sum_{i=0}^{n-1} \alpha^{m i}(v) .
$$

Then $w^{\prime}$ is close to a partial isometry and we will obtain a partial isometry $w$ in a small neighbourhood of $w^{\prime}$ such that $w^{*} w=1-e, w w^{*} \leq e_{0}, w$ is almost invariant under $\alpha^{m}$ (if $n$ is large), and $w$ is almost central (if $B$ is large). Then the $C^{*}$-algebra $D$ generated by $w, \alpha(w), \ldots, \alpha^{m-1}(w)$ is isomorphic to $M_{m+1}$, and $\alpha \mid D$ is close to $\operatorname{Ad} U \mid D$, where $U$ is a unitary in $D$ whose eigenvalues are

$$
1, e^{2 \pi i j / m} ; j=0,1, \ldots, m-1 .
$$


Since the eigenvalues of $U$ are almost uniformly distributed (if $m$ is large), one can find projections $f_{0}, \ldots, f_{l-1} ; g_{0}, \ldots, g_{l}$ in $D$ (for a prescribed $l$ ) such that they add up to the identity of $D$ and

$$
\alpha\left(f_{i}\right) \approx f_{i+1} ; \alpha\left(g_{i}\right) \approx g_{i+1}
$$

where $f_{l}=f_{0}$ and $g_{l+1}=g_{0}$. Hence the projections

$$
f_{0}, \ldots, f_{l-1} ; g_{0}, \ldots, g_{l} ; e_{0}-w w^{*}, \ldots, e_{m-1}-\alpha^{m-1}\left(w w^{*}\right)
$$

satisfy the required properties.

\section{Outer conjugacy}

Definition 5.1 Let $S_{k}$ be a finite sequence $\left\{s_{k 1}, \ldots, s_{k a_{k}}\right\}$ in $\mathbf{T}$ for each $k \in \mathbf{N}$. The sequence $\left\{S_{k}\right\}$ is uniformly distributed on $\mathbf{T}$ if for any continuous function $f$ on $\mathbf{T}$

$$
\lim _{k} a_{k}{ }^{-1} \sum_{i=1}^{a_{k}} f\left(s_{k i}\right)=\int_{\mathbf{T}} f(\lambda) d \lambda
$$

where $d \lambda$ is normalized Haar measure on $\mathbf{T}$.

See [4] for other equivalent conditions.

Lemma 5.2 Let $\alpha$ be an automorphism of a UHF algebra $A$ such that $\alpha=\otimes_{k=1}^{\infty} A d u_{k}$ on $A=\otimes_{k=1}^{\infty} M_{m_{k}}$, where $u_{k}$ is a unitary in the $k$-th factor $M_{m_{k}}$. For $k, l \in \mathbf{N}$ with $l \leq k$ let $S_{l, k}$ be a sequence consisting of the eigenvalues of the unitary $\otimes_{i=l}^{k} u_{i}$ repeated as often as multiplicity indicates. Then $\alpha$ has the Rohlin property if and only if $\left\{S_{l, k}\right\}_{k=l}^{\infty}$ is uniformly distributed for any $l \in \mathbf{N}$.

Proof. This is almost contained in [14,4]. Suppose that $\alpha$ has the Rohlin property, and that a sufficiently large $N \in \mathbf{N}$ is given for $k$ in Definition 1:1. Then there exist $N_{1}, \ldots, N_{t} \geq N$ such that for any $\epsilon>0$ there are projections $e_{i j} ; i=1, \ldots, t, j=$ $0, \ldots, N_{i}-1$ which are adding up to 1 and satisfy that $\left\|\alpha\left(e_{i, j}\right)-e_{i, j+1}\right\|<\epsilon$, where $e_{i, k_{i}}=e_{i, 0}$. This shows that for a sufficiently large $k \in \mathrm{N}$, the unitary $\bigotimes_{i=1}^{k} u_{i}$ is almost unitarily equivalent to

$$
\oplus_{i=1}^{t} U_{i} \otimes V_{i}
$$

where $U_{i}$ is a unitary of $M_{N_{i}}$ with eigenvalues

$$
e^{2 \pi i j / N_{i}} ; j=0, \ldots, N_{i}-1
$$

and $V_{i}$ is a unitary of $M_{m_{1} \cdots m_{k} \tau_{i}}$ with $\tau_{i}=\tau\left(e_{i 0}\right)$. This shows that the eigenvalues of $\otimes_{i=1}^{k} u_{i}$ is almost uniformly distributed whatever $V_{i}$ 's are, i.e., $\left\{S_{1, k}\right\}$ is uniformly distributed. This argument applies to the restriction of $\alpha$ to the subalgebra $\otimes_{k=l}^{\infty} M_{m_{k}}$ of $A$ for any $l \in \mathbf{N}$, concluding that $\left\{S_{l, k}\right\}_{k=l}^{\infty}$ is uniformly distributed. 
Suppose that $\left\{S_{l, k}\right\}_{k=l}^{\infty}$ is uniformly distributed for any $l \in N$. Then it is easy to construct the required projections as in Definition 1.1 with $F=\emptyset$ in $\otimes_{i=l}^{k} M_{m_{i}}$ for a sufficiently large $k$. (This is a linear algebra problem.) Hence the Rohlin property follows (see $[4,16]$ for details).

When $\left\{S_{l, k}\right\}_{k=l}^{\infty}$ is uniformly distributed in Lemma 5.2, we say that the eigenvalues of $\otimes_{i=l}^{\infty} u_{i}$ are uniformly distributed.

Proof of Theorem 1.4. As noted in Section 1, if $\alpha$ and $\beta$ have the Rohlin property, for any $\epsilon>0$ there exist unitaries $u_{1}, u_{2} \in A$ such that $\left\|u_{1}-1\right\|<\epsilon,\left\|u_{2}-1\right\|<\epsilon$ and $\operatorname{Ad} u_{1} \circ \alpha$ and $\operatorname{Ad} u_{2} \circ \beta$ are of infinite tensor product type. Hence we may assume that there is a sequence $\left\{m_{k}\right\}$ of positive integers such that $(A, \alpha)$ is isomorphic to

$$
\left(M_{m_{1}} \otimes \bigotimes_{i=1}^{\infty}\left(M_{m_{2 i}} \otimes M_{m_{2 i+1}}\right), \bigotimes_{i=0}^{\infty} \mathrm{Ad} u_{2 i}\right)
$$

where $u_{2 i} \in M_{m_{2 i}} \otimes M_{m_{2 i+1}}$, with $M_{m_{0}}=\mathrm{C}$ and $(A, \beta)$ is isomorphic to

$$
\left(\bigotimes_{i=1}^{\infty}\left(M_{m_{2 i-1}} \otimes M_{m_{2 i}}\right), \bigotimes_{i=0}^{\infty} \mathrm{Ad} u_{2 i-1}\right)
$$

where $u_{2 i-1} \in M_{m_{2 i-1}} \otimes M_{m_{2 i}}$. Since the eigenvalues of $\otimes_{i=1}^{\infty} u_{2 i-1}$ are uniformly distributed, it follows that for a sufficiently large $k, \otimes_{i=1}^{k} u_{2 i-1}$ is almost equal to $u_{0} \otimes V$ in norm (up to conjugacy) where $V$ is a unitary in $M_{m_{2} \cdots m_{2 k}}$. Then replacing $M_{m_{2}}$ by $M_{m_{2} \cdots m_{2 k}}$ we may assume that there is a unitary $w_{1} \in M_{m_{1}} \otimes M_{m_{2}}$ such that $\left\|w_{1}-1\right\|<\epsilon / 2, w_{1} u_{1}=u_{0} \otimes v_{2}$ where $v_{2}$ is a unitary of $M_{m_{2}}$. Note that $(A, \alpha)$ is still of the same form. In the same way we may assume that there is a unitary $w_{2} \in M_{m_{2}} \otimes M_{m_{3}}$ such that $\left\|w_{2}-1\right\|<$ $\epsilon / 2^{2}, w_{2} u_{2}=v_{2} \otimes v_{3}$ where $v_{3}$ is a unitary of $M_{m_{3}}$. Repeating this procedure we obtain unitaries $w_{i} \in M_{m_{i}} \otimes M_{m_{i+1}}$ and $v_{i} \in M_{m_{i}}$ such that

$$
\begin{aligned}
& \left\|w_{2 i}-1\right\|<2^{-i} \epsilon, \\
& w_{i} u_{i}=v_{i} \otimes v_{i+1}
\end{aligned}
$$

for $i=1,2, \ldots$ where $v_{1}=u_{0}$. Let

$$
w_{e}=\otimes_{i=0}^{\infty} w_{2 i}, w_{o}=\otimes_{i=0}^{\infty} w_{2 i-1} .
$$

Then $w_{e}, w_{o}$ are unitaries of $A$ with $\left\|w_{e}-1\right\|<\epsilon,\left\|w_{o}-1\right\|<\epsilon$ and

$$
\begin{aligned}
& \operatorname{Ad} w_{e} \circ \alpha=\otimes_{i=0}^{\infty} \operatorname{Ad} w_{2 i} u_{2 i}=\bigotimes_{i=0}^{\infty} \operatorname{Ad} v_{i}, \\
& \operatorname{Ad} w_{\circ} \circ \beta=\otimes_{i=0}^{\infty} \operatorname{Ad~} w_{2 i-1} u_{2 i-1}=\bigotimes_{i=0}^{\infty} \operatorname{Ad} v_{i} .
\end{aligned}
$$

This completes the proof. 


\section{Non-commutative tori}

Let $\Theta=\left(\theta_{i j}\right) \in M_{n}(\mathbf{R})$ be such that $\theta_{i j}=-\theta_{j i}$ and let $A_{\Theta}$ be the universal $C^{*}$-algebra generated by $n$ unitaries $u_{1}, \ldots, u_{n}$ with relations $u_{i} u_{j} u_{i}^{*} u_{j}^{*}=e^{2 \pi i \theta_{i j}} 1 ; A_{\Theta}$ is called a noncommutative torus. We call $\Theta$ to be completely irrational if for any $x \in \mathbf{Z}^{n} \backslash\{0\}$ there is a $y \in \mathbf{Z}^{n}$ with $(\Theta x, y) \notin \mathbf{Z}$, i.e., $u^{x}=u_{1}^{x_{1}} \cdots u_{n}^{x_{n}}$ is not in the center of $A_{\Theta}$. It follows that $A_{\Theta}$ is simple if and only if $\Theta$ is completely irrational. In this case $A_{\Theta}$ has a unique tracial state. Define an action $\alpha$ of $\mathbf{T}^{n}$ on $A_{\Theta}$ by $\alpha_{\lambda}\left(u_{i}\right)=\lambda_{i} u_{i}$ with $\lambda=\left(\lambda_{1}, \ldots, \lambda_{n}\right)$. The action $\alpha$ is ergodic and the above tracial state is defined as the average over this action. For a $\lambda \in \mathbf{T}^{n} \alpha_{\lambda}$ is inner if and only if $\lambda_{j}=e^{2 \pi i(\Theta x)_{j}}$ for some $x \in \mathbf{Z}^{n}$, i.e., $\alpha_{\lambda}$ is implemented by $u^{x}$ for some $x \in \mathbf{Z}^{n}$.

Theorem 6.1 Let $A_{\Theta}$ be as above and suppose that $A_{\Theta}$ is a simple $C^{*}$-algebra. Let $\lambda \in \mathrm{T}^{n}$ be such that $\alpha_{\lambda^{m}}$ is outer for any $m \neq 0$. Then $\alpha_{\lambda}$ has the Rohlin property.

Proof. We may assume that $\theta_{12}$ is irrational and $\lambda_{1}^{m} \notin\left\{e^{2 \pi i(\Theta x)_{1}} ; x \in \mathbf{Z}^{n}\right\}$ for all $m \neq 0$. Let $\rho=e^{2 \pi i \theta_{12}}$. Then, by using the proof of Lemma 4.6 of [3], for any $\epsilon>0$ there are $p, q \in \mathbf{N}$ such that

$$
\begin{aligned}
& \left|\rho^{p}-1\right|,\left|\lambda_{1}^{p}-\lambda_{1}\right|,\left|e^{2 \pi i p \theta_{k 1}}-1\right|(k=3, \ldots, n), \\
& \left|\rho^{q}-1\right|,\left|\lambda_{2}^{q}-1\right|,\left|e^{2 \pi i q \theta_{k 1}}-1\right|(k=3, \ldots, n), \\
& \left|\rho^{p q}-\rho\right|
\end{aligned}
$$

are all smaller than $\epsilon$. (First choose a sufficiently large $p$ such that the values in the first line above are all smaller than $\epsilon$, and then choose $q$ such that $\left|\rho^{q}-\rho^{1 / p}\right|,\left|\lambda_{2}^{q}-1\right|,\left|e^{2 \pi i q \theta_{k 1}}-1\right|$ are sufficiently small, where $\rho^{1 / p}=e^{2 \pi i \theta_{12} / p}$. Then the estimate on $\left|\rho^{q}-\rho^{1 / p}\right|$ will yield both $\left|\rho^{q}-1\right|<\epsilon$ and $\left|\rho^{p q}-\rho\right|<\epsilon$.) For $\epsilon=1 / m$ let $v_{m}=u_{1}^{p}$ and $w_{m}=u_{2}^{q}$. Then $\left\{v_{m}, w_{m}\right\}$ forms a central sequence of pairs of unitaries in $A_{\Theta}$ such that

$$
\begin{aligned}
& v_{m} w_{m} v_{m}^{*} w_{m}^{*}=\rho_{m} 1, \rho_{m} \rightarrow \rho, \\
& \alpha_{\lambda}\left(v_{m}\right)=\mu_{m} v_{m}, \mu_{m} \rightarrow \lambda_{1}, \\
& \alpha_{\lambda}\left(w_{m}\right)=\nu_{m} w_{m}, \nu_{m} \rightarrow 1
\end{aligned}
$$

By applying Lemma 6.3 below we can conclude the proof.

Lemma 6.2 Let $\theta$ be an irrational number and let $A_{\theta}$ be the non-commutative torus corresponding to $\Theta \in M_{2}(\mathbf{R})$ with $\theta_{12}=\theta$. Let $\lambda \in \mathbf{T}^{2}$ be such that any non-zero power of $\alpha_{\lambda}$ is outer. Then $\alpha_{\lambda}$ has the approximate Rohlin property in the sense that for any $m \in \mathbf{N}$ there is a central sequence of orthogonal families $\left\{e_{i}^{(j)} ; i=0, \ldots, m-1\right\}$ of projections in $A_{\theta}$ such that

$$
\begin{aligned}
& \left\|\alpha_{\lambda}\left(e_{i}^{(j)}\right)-e_{i+1}^{(j)}\right\| \rightarrow 0, \\
& \tau\left(e_{0}^{(j)}\right) \rightarrow 1 / m
\end{aligned}
$$

where $e_{m}^{(j)}=e_{0}^{(j)}$ and $\tau$ is the unique tracial state of $A_{\theta}$. 
Proof. Note that $A_{\theta}$ is a nuclear $C^{*}$-algebra of real rank zero [3] and that $K_{\mathbf{0}}\left(A_{\theta}\right) \cong \mathbf{Z}+\theta \mathbf{Z}$ and $A_{\theta}$ has cancellation [1]. We can use the proof of Lemma 4.3 to obtain such a sequence if we drop the requirement of centralness. We needed the AF property there to make the sequence central; othewise the above mentioned properties would suffice. To obtain a central sequence of such families, we use the argument in the proof of Theorem 6.1; assuming that $(2 \pi)^{-1} \log \lambda_{1}$ is rationally independent of $\theta$, we obtain a central sequence $\left\{v_{k}, w_{k}\right\}$ of pairs of unitaries in $A_{\theta}$ such that

$$
\begin{aligned}
& v_{k} w_{k} v_{k}^{*} w_{k}^{*}=\rho_{k} 1, \rho_{k} \rightarrow e^{2 \pi i \theta}, \\
& \alpha_{\lambda}\left(v_{k}\right)=\mu_{k} v_{k}, \mu_{k} \rightarrow \lambda_{1}, \\
& \alpha_{\lambda}\left(w_{k}\right)=\nu_{k} w_{k}, \nu_{k} \rightarrow 1 .
\end{aligned}
$$

On the other hand, for any $\epsilon>0$ and $m \in \mathbf{N}$ we have an orthogonal family $\left\{e_{i} ; i=\right.$ $0, \ldots, m-1\}$ of projections in $A_{\theta}$ such that

$$
\begin{aligned}
& \left\|\alpha_{\left(\lambda_{1}, 1\right)}\left(e_{i}\right)-e_{i+1}\right\|<\epsilon, \\
& m \tau\left(e_{0}\right)>1-\epsilon,
\end{aligned}
$$

where $e_{m}=e_{0}$. Then we obtain a continuous field of such families $\left\{e_{i}(t) ; i=0, \ldots, m-1\right\}$ in $A_{t}$ with $t$ in a small neighbourhood of $\theta$ [11]. Since the action $\alpha$ of $\mathbf{T}^{2}$ acts continuously on this field, we have that for $t$ in a small neighbourhood of $\theta$ and for $\mu$ in a small neighbourhood of $\left(\lambda_{1}, 1\right)$,

$$
\begin{aligned}
& \left\|\alpha_{\mu}\left(e_{i}(t)\right)-e_{i+1}(t)\right\|<\epsilon, \\
& m \tau\left(e_{i}(t)\right)>1-\epsilon,
\end{aligned}
$$

where $e_{m}(t)=e_{0}(t)$. By identifying the $C^{*}$-algebra $B_{k}$ generated by $v_{k}, w_{k}$ with $A_{\theta_{k}}$ with $\rho_{k}=e^{2 \pi i \theta_{k}}$, we obtain the family $\left\{e_{i}\left(\theta_{k}\right)\right\}$ of projections in $B_{k}$. Since they form a central sequence, we can now conclude the proof.

Lemma 6.3 Let $A_{\theta}, \alpha_{\lambda}$ be as in Lemma 6.2. Then for any $k \in \mathbf{N}$ and any $\epsilon>0$ there are $k_{1}, \ldots, k_{m} \geq k$ and projections $e_{i, j} ; i=1, \ldots, m, j=0, \ldots, k_{i}-1$ in $A_{\theta}$ such that

$$
\begin{aligned}
& \sum_{i=1}^{m} \sum_{j=0}^{k_{i}-1} e_{i, j}=1, \\
& \left\|\alpha_{\lambda}\left(e_{i, j}\right)-e_{i, j+1}\right\|<\epsilon,
\end{aligned}
$$

where $e_{i, k_{i}}=e_{i, 0}$.

Proof. This can be proved as Lemma 4.4, by using the fact that if two projections in $A_{\theta}$ have the same trace value then they are equivalent. Furthermore, in Definition 1.1 of the Rohlin property, we can choose $m=2, k_{1}=k, k_{2}=k+1$ as in the case of UHF algebras (cf. [17]). This follows by noting that we can choose, in Lemma 6.2 , matrix units $\left\{e_{i, k}^{(j)}\right\}$ such that $\left\|\alpha_{\lambda}\left(e_{i, k}^{(j)}\right)-e_{i+1, k+1}^{(j)}\right\|<\epsilon, \tau\left(e_{0,0}\right) \rightarrow 1 / m$ instead of just families of projections (see the proof of Theorem 2.1 in [15] for details). 


\section{References}

[1] B. Blackadar, K-theory for Operator Algebras, MSRI Publication series 5, SpringerVerlag, 1986.

[2] B. Blackadar, O. Bratteli, G.A. Elliott, and A. Kumjian, Reduction of real rank in inductive limits of $C^{*}$-algebras, Math. Ann. 292(1992), 111-126.

[3] B. Blackadar, A. Kumjian, and M. Rørdam, Approximately central matrix units and the structure of non-commutative tori, K-Theory 6(1992), 267-284.

[4] O. Bratteli, D.E. Evans, and A. Kishimoto, The Rohlin property for quasi-free automorphisms of the Fermion algebra, Proc. London Math. Soc. (to appear)

[5] O. Bratteli, D.E. Evans, and A. Kishimoto, Almost shift invariant projections in infinite tensor products, in Quantum and Non-Commutative Analysis H. Araki et al eds. Kluwer, 427-434, 1993.

[6] O. Bratteli, A. Kishimoto, M. Rørdam, and E. Størmer, The crossed product of a UHF algebra by a shift, Ergod. Th. \& Dynam. Sys. 13(1993), 615-626.

[7] L. Brown and G.K. Pedersen, $C^{*}$-algebras of real rank zero, J. Func. Anal. 99(1991), 131-149.

[8] A. Connes, Periodic automorphisms of hyperfinite factors of type $\mathrm{II}_{1}$, Acta Sci. Math. 39(1977), 39-66.

[9] A. Connes, Outer conjugacy class of automorphisms of factors, Ann. Scient. Ec. Norm. Sup., $4^{e}$ serie, 8(1975), 383-420.

[10] E.G. Effros, Dimensions and $C^{*}$-algebras, CBMS regional conference series in math. 46, 1981.

[11] G.A. Elliott, On the K-theory of the $C^{*}$-algebra generated by a projective representation of a torsion free abelian group, in Operator Algebra and Group Representation, vol 1, Pitman, 157-184, 1983.

[12] G.A. Elliott, On the classification of $C^{*}$-algebras of real rank zero, J. reine angew. Math. 443(1993), 179-219.

[13] R.H. Herman and A. Ocneanu, Stability for integer actions on UHF $C^{*}$-algebras, J. Funct. Anal. 59(1984), 132-144.

[14] R.H. Herman and A. Ocneanu, Spectral Analysis for automorphisms of UHF $C^{*}$ algebras, J. Funct. Anal. 66(1986), 1-10. 
[15] A. Kishimoto, The Rohlin property for shifts on UHF algebras and automorphisms of Cuntz algebras, preprint.

[16] A. Kishimoto and A. Kumjian, Crossed products of Cuntz algebras by quasi-free automorphisms, preprint.

[17] M. Rørdam, Classification of certain infinite simple $C^{*}$-algebras, J. Funct. Anal. (to appear)

[18] D. Voiculescu, Almost inductive limit automorphisms and embeddings into AFalgebras, Ergod, Th. \& Dynam. Sys. 6(1986), 475-484.

Department of Mathematics, Hokkaido University, Sapporo 060 Japan 